\title{
Contaminación de los ambientes acuáticos generados por la industria textil
}

Pollution of aquatic environments generated by textile industry

Recibido: julio 12 de 2018 | Revisado: agosto 13 de 2018 | Aceptado: setiembre 10 de 2018

\author{
Marco Brañez SÁncheZ \\ RÓMULO GuTIÉRREZ \\ Ronald PÉrez \\ Carmen Uribe \\ Pamela Valle
}

\begin{abstract}
RESUMEN
La industria textil es, después de la industria del petróleo, la segunda industria más contaminante del medio ambiente y sobre todo, de la que no se tiene tanta conciencia. El sector textil y confecciones es un negocio complejo ya que involucra largas y variadas cadenas de suministro de producción, materias primas, fabricación de textiles, diseño de prendas de vestir, envío, ventas, uso y finalmente, la disposición final de la prenda. En 2015 la población mundial ha alcanzado los 7.400 millones de personas y el consumo textil per cápita llegó a los 13,1 $\mathrm{kg} / \mathrm{hab} / \mathrm{anno}$. La cantidad de agua necesaria para fabricar las prendas que vestimos varían de acuerdo a la fibra textil y colorante usados: Un par de jean de algodón con colorantes índigos, necesita un mínimo de 42 litros de agua para ser teñidos, lavados y acabados. Una vez en casa, empleamos más agua, cuidándolos; usando, 21 litros cada vez que los lavamos. La industria textil tiene como principales impactos ambientales el alto consumo del recurso agua y las aguas residuales con alta carga contaminante que se generan en los diversos procesos. En la investigación se revisó la problemática de la industria textil, específicamente en el ramo del agua y los diversos enfoques para reducir la contaminación del agua que van desde la reducción en la fuente, recuperación (reuso y reciclaje), tratamiento centralizado de efluentes y separación de ciertos baños de proceso para tratamiento individual o reutilización.
\end{abstract}

Palabras claves: Industria textil, contaminación del agua, aguas residuales, tratamiento, colorantes textiles

\begin{abstract}
The textile industry is, right behing the oil industry, the second most polluting industry in the environment and, above all, one of which we do not have as much awareness. The textile and clothing sector is a complex business since it involves long and varied supply chains of production, raw materials, textile manufacturing, garment design, shipping, sales, use and finally, the final disposal of the garment. In 2015, the world population reached 7.4 billion people and textile consumption per capita reached $13.1 \mathrm{~kg} /$ inhabitant / year. The amount of water needed to manufacture the garments we wear varies according to the textile fiber and dye used: A pair of cotton jeans with indigo dyes need a minimum of 42 liters of water to be dyed, washed and finished. Once at home, we use more water taking care of them; using,
\end{abstract}

Escuela Universitaria de Posgrado

UNFV, Lima-Perú

Maestría en Gestión Ambiental

mbranezs@uni.edu.pe

curibe@uni.edu.pe 
21 liters each time we wash them. The main environmental impacts of the textile industry are the high consumption of water resources and the wastewater with a high polluting load that are generated in the various processes. In this research we review the problems of the textile industry, specifically in the field of water and the various approaches to reduce water pollution ranging from source reduction, recovery (reuse and recycling), centralized treatment of effluents and separation of certain process baths for individual treatment or reuse.

Key words: Textile industry, water pollution, wastewater, treatment, textile dyes

\section{Introducción}

La industria consume entre 5 a $20 \%$ del agua disponible, y a su vez genera una significativa parte de la contaminación de las aguas (WWAP Programa Mundial de Evaluación de los Recursos Hídricos, 2009). La presión social y medioambiental dio lugar a un creciente movimiento que instó a la industria a reducir la cantidad de aguas residuales que produce y a tratarla antes de su eliminación. Esto ha convertido a las aguas residuales en un potencial recurso y a su uso o reciclaje, después de un tratamiento adecuado, en una posibilidad de beneficiar a la industria financiera y económicamente (WWAP-Programa Mundial de Evaluación de los Recursos Hídricos de las $\mathrm{Na-}$ ciones Unidas, 2017).

En Perú, la magnitud real del volumen de las aguas residuales que las industrias formales e informales producen no es conocida con exactitud y a escala mundial los datos e información que se tienen son escasos. A nivel mundial, una estimación sugiere que los volúmenes de aguas residuales industriales se duplicarán para el 2025. Se prevé que para ese año un total de 1800 millones de personas vivirán en países o regiones aquejadas por escasez de agua (Iniciativa Financiera del Progra- ma de las Naciones Unidas para el Medio Ambiente - UNEP FI, 2007).

La industria textil se caracteriza por ser una de las actividades que tiene altos consumos de: agua, energía y reactivos químicos. Cada planta no usa el mismo proceso de producción, ni químicos y tampoco usa la misma cantidad de agua. En 2015, la población ha alcanzado los 7400 miles de millones de personas y el consumo textil per cápita llegó a los 13,1 $\mathrm{kg} / \mathrm{hab} / \mathrm{año} \mathrm{(Carrera,} \mathrm{2016).} \mathrm{A} \mathrm{continua-}$ ción, datos importantes que resaltar de esta industria:

- Se consumen 54 millones de toneladas de fibras naturales/Año, un millón toneladas de colorantes y siete millones de químicos para procesamiento textil/Año.

- El algodón es la fibra que más consume agua en su proceso de tintura: 30 - $150 \mathrm{~L} / \mathrm{kg}$ de material textil (Castells, 2012).

- Un par de jeans necesita un mínimo de 42 litros de agua para ser teñidos, lavados y acabados. Una vez en casa, usamos todavía más agua, cuidándolos; usando, 21 litros cada vez que los lavamos (Villegas y Gonzales, 2013).

- Por cada kilo de producto acabado (prenda) se emite 3,6 kg de $\mathrm{CO}_{2}$ 
equivalente (Salas y Condorhuaman, 2014)

En la Figura 1 se muestra una curva típica de proceso de teñido por agota-

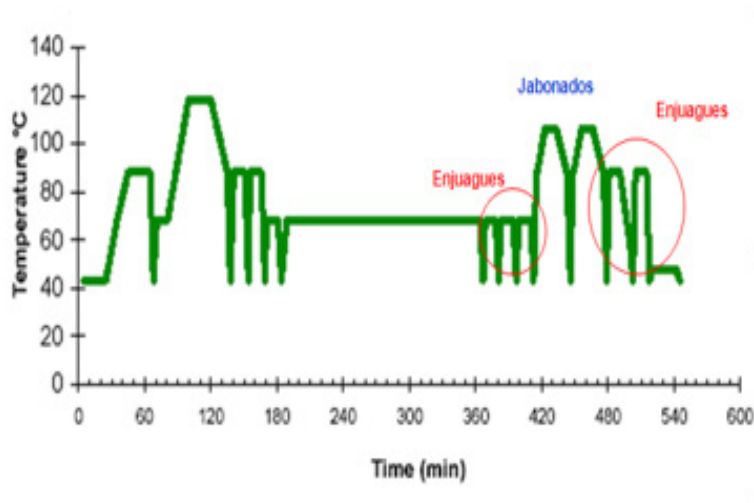

Figura 1. Curva y teńido de algodón.

Fuente: Elaboración propia

La industria textil se caracteriza también por ser una actividad que genera más residuos contaminantes. El agua residual generada por los diversos procesos textiles tiene que ser limpia de: grasas, aceites, colorantes y otros productos químicos antes de su descarga al alcantarillado.

Es así que para tener procesos textiles sustentables necesitamos:

- Minimizar la contaminación: aire, agua y suelo. miento (temperatura versus tiempo) y la maquinaria para el teńido de tejidos de punto. Se puede apreciar el alto consumo de agua en 14 baños en un tiempo total de 550 minutos.

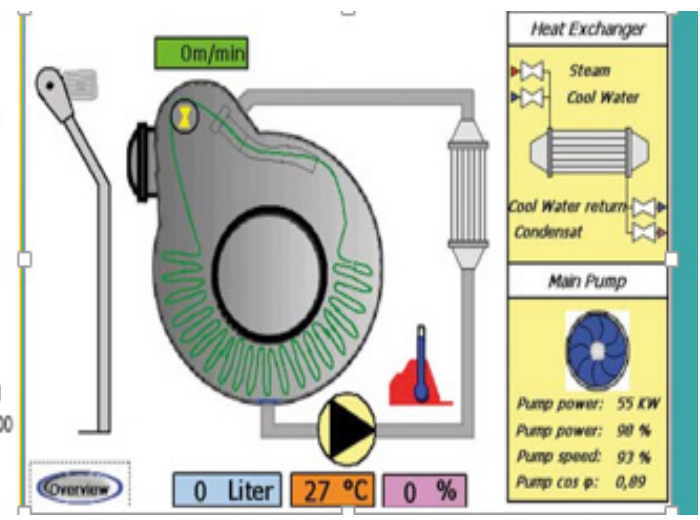

- Optimizar los recursos: energía, agua, tiempo, químicos.

- Garantizar la seguridad del trabajador y el consumidor.

Por tanto, para que los procesos textiles sean verdes necesitamos:

- Uso mínimo de energía: menor huella de carbono

- Uso mínimo de agua: menor huella hídrica

- Minimizar la contaminación de descargas: ríos y efluentes limpios.

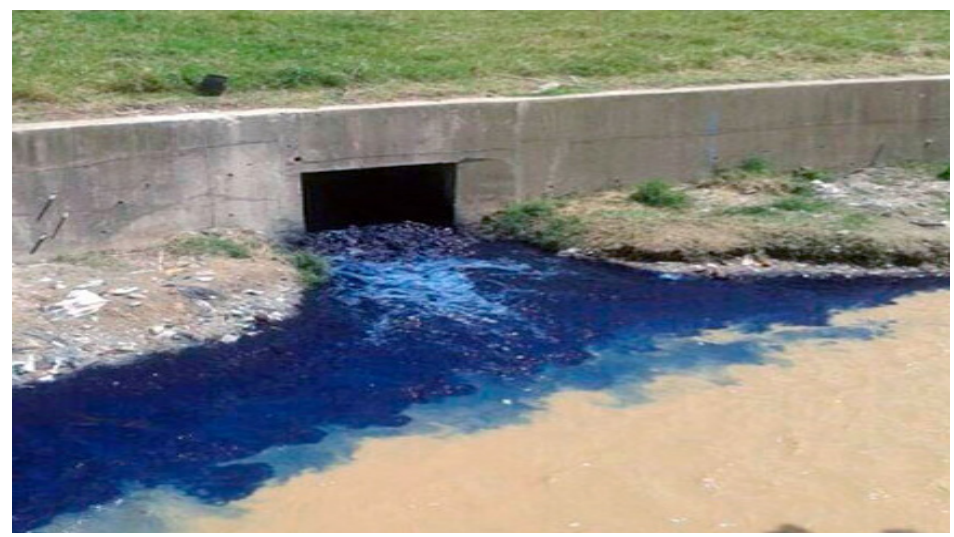

Figura 2. Descarga de aguas residuales textiles a un río.

Fuente:http://agenciadenoticias.unal.edu.co/detalle/article/cascarilla-de-arroz-para-remover-colorantes.htm 
Las aguas residuales textiles como se muestra en la Figura 2 tienen altas concentraciones de colorantes con toxicidad acuática. El proceso de tratamiento de efluentes seleccionado dependerá de las características de las aguas residuales. Un químico seguro usado inadecuadamente es más contaminante que un químico dańino usado adecuadamente. El objetivo no es otro que empujar a la industria textil hacia una cadena de suministros con mayor transparencia.

\section{Materiales y métodos}

Para este trabajo, se revisaron artículos de investigación de las bases de datos Scielo y Scopus. También se analizaron tesis de investigación de los repositorios académicos de universidades peruanas, de Latinoamérica y Europa. A través del Google Académico accedimos a publicaciones realizadas en revistas científicas además de manuales, normas y guías procedimentales.

Toda la información obtenida pertenece al periodo comprendido entre los ańos 1998 al 2017 y fueron el resultado de búsquedas con palabras clave: Contaminación del agua, agua residual, industria textil, colorantes textiles, entre otras.

Se obtuvo un total de 26 documentos de los cuales el 40,6\% fue de Latinoamérica, 55.4\% de Europa y 4,0\% de Estados Unidos de Norteamérica. Destacan en la pesquisa Colombia y México en Latinoamérica y España en Europa. Con la información recopilada, cada investigador revisó y analizó la bibliografía referida al tema elegido para la investigación y luego el equipo de trabajo en conjunto concluyó en los resultados y discusión.

\section{Resultados}

\section{El agua en la industria textil}

\section{Características del agua}

Fórmula: H2O. Densidad: 1,00000 a $4^{\circ} \mathrm{C} 0,95838$ a $100^{\circ} \mathrm{C}$

Calor latente: $2,250 \mathrm{~kJ} / \mathrm{kg}$, por eso se le usa como fluido para transferir calor.

Característica oxidante: $\mathrm{E}^{\circ}=0,828$ voltios

Característica reductora: $\mathrm{E}^{\circ}=-0,401$ voltios

El agua es más fácil de reducir que oxidar.

\section{Polaridad}

Las moléculas del agua están dobladas con un ángulo de enlace de $104,5^{\circ}$ y debido a esta geometría la distribución de la carga dentro de la molécula es asimétrica y por tanto es polar. Esta característica es la que permite la solubilidad de los colorantes en polvo que se usan en la industria textil.

\section{Tensión superficial del agua}

Es elevada por su estructura y su cohesión entre las moléculas por los "puentes de hidrógeno". Su valor decrece al aumentar la temperatura.

\section{Puentes de hidrógeno}

Son los responsables de la gran cohesión, o atracción mutua, de las moléculas de agua. La cohesión trae como consecuencia la alta tensión superficial, la cual es necesario bajar en los procesos textiles con temperatura o con la adición de un agente tensoactivo y así lograr penetrar las fibras naturales, sintéticas y regeneradas.

\section{Agua dura}

Agua que contiene más minerales de calcio y magnesio. El grado de dureza 
aumenta cuanto más calcio y magnesio haya disuelto. Se mide en $\mathrm{mg} / \mathrm{L}$ o ppm de $\mathrm{CaCO}_{3}$

\section{Agua blanda}

Es el agua libre de sales de calcio y magnesio. Se puede obtener tratando al agua dura de pozo de las empresas con sistemas de intercambio iónico, que eliminan los iones (cationes y aniones) que contiene el agua (Figura 3). Generalmente, para la industria textil se usa solo el intercambio catiónico utilizando zeolita de sodio. Los ablandadores de agua son específicos intercambiadores de iones que son diseñados para eliminar iones, un ejemplo es el calcio y el magnesio $\left(\mathrm{Ca}^{+2}\right)\left(\mathrm{Mg}^{+2}\right)$, que se encuentran en el agua dura.

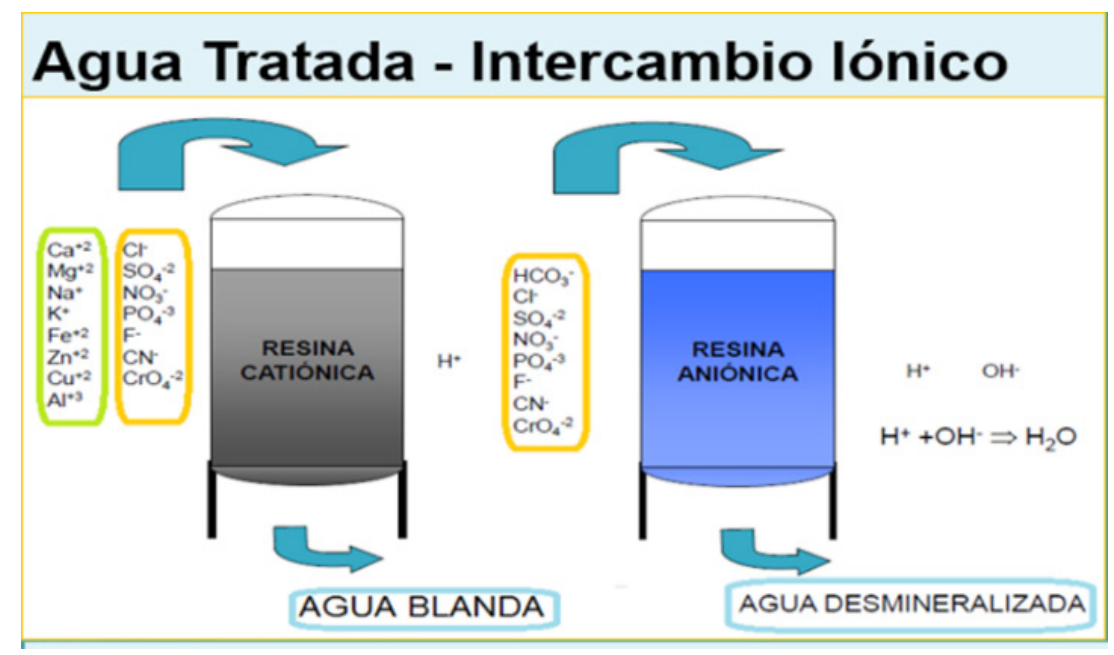

Figura 3. Agua tratada por intercambio iónico.

Fuente: López (2013)

\section{Procesos químicos textiles}

Los procesos químicos textiles incluyen todas aquellas operaciones en las que la materia textil se somete a un tratamiento químico (Figura 4) con la finalidad de que mejoren su aspecto, perfeccionen su comportamiento al uso o que su cuidado sea más fácil. En todas ellas los productos químicos son transportados a través de agua por lo que este proceso también se le conoce como: ramo del agua.

\section{Proceso de preparación}

Como su propio nombre lo indica, la preparación es la etapa donde se adecuará el textil en crudo y de acuerdo al tipo de fibra, matiz y colorante estará compuesto el total de operaciones de purificación de los materiales textiles. La purificación o limpieza depende, primordialmente, del tipo de fibra (naturales, sintéticas o regeneradas) y existen diferentes maneras de hacerlo.

Las fibras naturales de origen vegetal o animal son las que contienen mayores impurezas y requieren de procesos de purificación más fuertes que las fibras manufacturadas (sintéticas o regeneradas). 


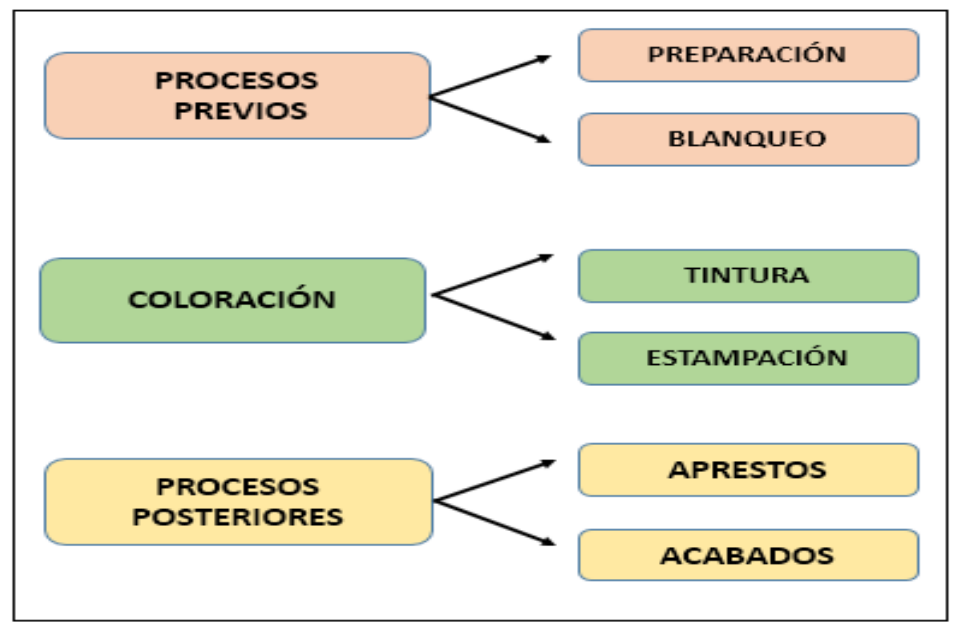

Figura 4. Procesos químicos textiles

Fuente: Elaboración Propia

Tenemos los siguientes procesos de purificación o limpieza:

- Lavado

- Desengomado (en tejido plano)

- Descrudado

- Blanqueo químico

\section{Procesos de teñido}

El teñido del textil es una de las fases más complejas del proceso productivo pues en ella intervienen una gran diversidad de colorantes y agentes auxiliares de teñido.

\section{Colorantes textiles}

El término colorante abarca todo compuesto que cuando es aplicado sobre un objeto le confiere color y que mantiene sus propiedades de color por un tiempo prolongado.

\begin{tabular}{|l|l|}
\hline FIBRAS CELULÓSICAS & Colorantes Directos \\
Naturales: Algodón & Colorantes Al Azufre \\
Regeneradas (artificiales): & Colorantes Tinas \\
Modal, Cupro, Tencel, & Colorantes Reactivos \\
Viscosa, etc. & \\
FIBRAS PROTÉICAS & Colorantes Ácidos \\
Naturales: Lana & Colorantes Dispersos \\
FIBRAS SINTÉTICAS & Colorantes Ácidos \\
\hline $\begin{array}{l}\text { Poliéster } \\
\text { Poliamida (Nylon) } \\
\text { Acrílico }\end{array}$ \\
\hline
\end{tabular}

Figura 5. Clasificación de los colorantes por su aplicación

Fuente: Elaboración propia

Cabe resaltar que el teñido es un proceso químico. Para que se produzca la atracción entre la fibra el colorante va a depender de la química de cada uno de ellos. En la Figura 5 se puede ver, claramente, que una fibra como el algodón se puede teñir con cuatro tipos de colorantes, así como el colorante disperso 
puede teñir a la poliamida (nylon) y al poliéster.

\section{Acabado textil}

Es el proceso complementario que se hace al textil para darle características adecuadas para su uso final. Generalmente, esto suele ser mejor tacto, así también sirve para mejorar algunas deficiencias que poseen las diferentes fibras, en especial el algodón que tiene una gran facilidad de arrugarse y encogerse.

Se entiende por acabado de un textil al tratamiento que recibe según el uso final a que vaya a ser destinado, y siempre para mejorar su aspecto y calidad. En la Figura 6, se muestra la Rama Tensora, máquina indicada para los acabados textiles. Pueden clasificarse en tres grupos:

1. Acabados generales: son aquellos a los que se someten los tejidos para obtener un determinado aspecto (limpieza, cepillado, secado, etc.).

2. Acabados con efecto de superficie: son aquellos que modifican la apariencia y el tacto de los tejidos originando uno nuevo. Suelen hacerse mediante procesos mecánicos o químicos (laminado, arrugado, etc.).

3. Acabados químicos: son aquellos que se dan a los artículos para mejorar su calidad y rendimiento, aunque su aspecto no cambie (antideslizante, antipilling, antiestático, anti moho).

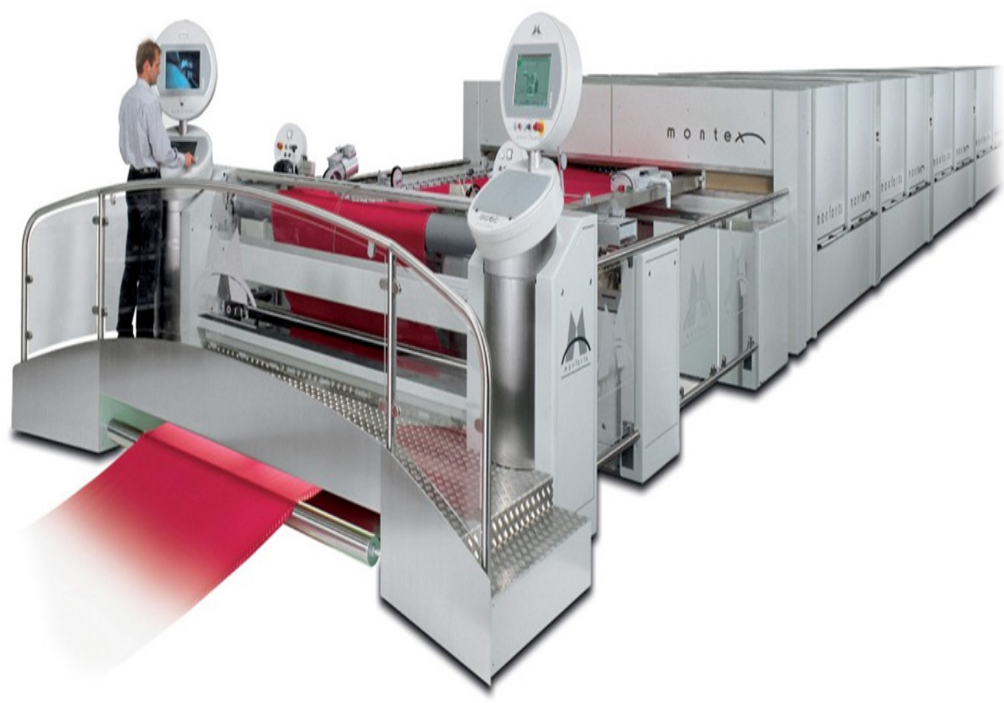

Figura 6. Rama tensora- máquina de acabado textil.

Fuente: Textiles Panamericanos (2005)

\section{Las aguas residuales}

Las características de las aguas residuales generadas en una planta textil dependerán de las operaciones específicas que se realicen, principalmente, del tipo de fibra tratada y de la maquinaria utilizada.
Las aguas residuales que se producen en la industria de fibras naturales, a excepción de las procedentes del lavado de lana, se caracterizan por una gran variabilidad de caudal y carga contaminante, así como un bajo contenido en materias coloidales y en suspensión. Comúnmente, 
son coloreadas, y su carga orgánica media es aproximadamente el doble que la de un agua residual urbana y no suelen ser tóxicas (Gutiérrez et al., 2006).

Los efluentes de fibras naturales se caracterizan por una gran variabilidad de caudal y de carga contaminante, así como un bajo contenido de materias en suspensión y coloidales. Generalmente son coloreadas, y su carga orgánica media es aproximadamente el doble que el de un agua residual urbana y no acostumbra a contener productos tóxicos ni microorganismos patógenos. Además, acostumbran a ser deficientes en nutrientes, principalmente en nitrógeno.

Dentro del sector textil, se encuentran dos grupos bien diferenciados de empresas según el curso receptor de sus vertidos: empresas que vierten sus aguas residuales a una red cloacal y empresas que las vierten a un curso de agua. Este segundo grupo de empresas que vierte directamente a un curso de agua debe cumplir con unos límites de vertido que de manera genérica acostumbran a ser más restrictivos que los límites de vertido a red cloacal (Gutierrez y Crespi, 2015).

El amplio uso que tienen los auxiliares en la industria textil ha hecho que se prestara atención a sus consecuencias ambientales desde hace mucho tiempo, especialmente, a la tendencia que tienen a la formación de espumas, al aumento de la carga biodegradable y a la contaminación con productos tóxicos observados en la descarga de los efluentes que los contienen.

Por estas razones, han ido surgiendo en diferentes comunidades restricciones a su uso y reglamentaciones que condicionan su vertido. En base a ello, la Subcomisión de Auxiliares Textiles decidió reunir los principales criterios involucrados en el uso de estos productos y su relación con el ambiente.

Muchos países han promulgado regulaciones que obligaron a las industrias a prestar atención al tema de contaminación ambiental. Sin embargo, las industrias no deben considerar el control de la contaminación como un sobrecosto sin retorno. De acuerdo a la definición de Boyston "la contaminación es un signo de ineficiencia en la producción industrial". Considera que la contaminación es dinero que se va por la chimenea, con los efluentes y en forma de residuos. La contaminación es, sobre todo, una descarga de materiales y energía en el ambiente.

En la evaluación ecológica de los productos para el acabado textil, incluyendo sus sustancias acompañantes (p.ej. formaldehído, monómeros, disolventes) se deben considerar los factores siguientes:

- La toxicidad durante la aplicación del producto, en el ambiente de trabajo.

- La contaminación de las aguas residuales

- Las emisiones atmosféricas durante el secado y la condensación.

- La influencia que pueda ejercer la prenda terminada en la salud del usuario.

\section{Contaminante emergente}

Es cualquier sustancia química sintética o natural o microorganismo que no se detecte comúnmente en el medio ambiente, pero que pueda entrar en él y cau- 
sar efectos adversos ecológicos y/o para la salud.

- Farmacéuticos (antibióticos, analgésicos, etc.)

- Productos de cuidado personal (fragancias, filtros UV, repelentes de insectos, micro perlas y antisépticos).
- Pesticidas, tensoactivos, retardantes de llama, plastificantes, etc.

En la Figura 7 se muestra cómo se producen las contaminaciones en las plantas textiles.

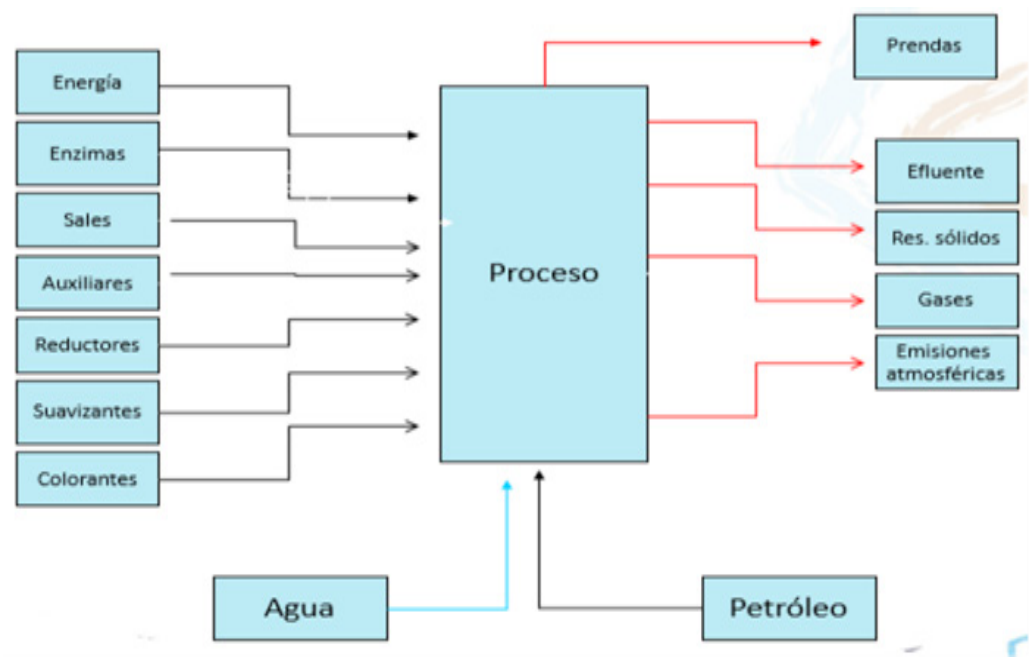

Figura 7. Flujo de la contaminación en las plantas textiles Fuente: Nava (2014)

Los colorantes sintéticos, actualmente, empleados masivamente en la industria textil presentan compuestos tóxicos con alto peso molecular los cuales al estar presentes en los efluentes industriales y acumularse en lagos y bahías provocan:

- La disminución en la luminosidad de las aguas

- La disminución de la actividad fotosintética

- Disminución del oxígeno disponible

- Aumento de carga orgánica

La mayoría de los colorantes tiene una toxicidad baja a los peces y organismos acuáticos. La generalidad de los colorantes básicos, los cuales comparten su emisión de toxicidad elevada con muchos materiales catiónicos es la excepción. Sin embargo, cuando son usados apropiadamente, los colorantes básicos se agotan casi completamente en la fibra; y por consiguiente, muy pequeñas cantidades de colorante, permanecen en el efluente final de una fábrica textil.

\section{Normativas Legales de las descargas de aguas residuales no domésticas}

En nuestro país el D.S. N021-2009

- Vivienda (Descargas de aguas residuales no domésticas en el sistema de alcantarillado sanitario), establece los estándares de valores máximos admisibles (VMA) de los efluentes. En la Tabla 1 se presenta los valores máximos admisibles de las descargas de aguas no domésticas en el sistema de alcantarillado sanitario. 
Tabla 1

Valores máximos admisibles de las descargas de aguas no domésticas en el sistema de alcantarillado sanitario (correspondientes a los anexos 1 y 2 del DS 021-2009).

\begin{tabular}{|c|c|}
\hline \multicolumn{2}{|c|}{ ANEXON'1 } \\
\hline Parámetro & VMA \\
\hline Demanda Bioquímica de Oxigeno (DBOs) & $500 \mathrm{mg} / \mathrm{t}$ \\
\hline Demanda Química de Oxigeno (DQO) & $1000 \mathrm{mg} / \mathrm{tt}$ \\
\hline Sólidos Suspendidos Totales & $500 \mathrm{mg} / \mathrm{tt}$ \\
\hline Aceites y Grasas & $100 \mathrm{mg} / \mathrm{th}$ \\
\hline
\end{tabular}

“ANEXO N"O2 (1)

\begin{tabular}{|l|c|c|c|}
\hline \multicolumn{1}{|c|}{ PARÁMETRO } & UNIDAD & EXPRESIÓN & $\begin{array}{c}\text { VMA PARA DESCARGAS } \\
\text { AL SISTEMA DE } \\
\text { ALCANTARILLADO } \\
\text { SANITARIO }\end{array}$ \\
\hline Aluminio & $\mathrm{mg} / \mathrm{L}$ & $\mathrm{Al}$ & 10 \\
\hline Arsénico & $\mathrm{mg} / \mathrm{L}$ & $\mathrm{As}$ & 0.5 \\
\hline Boro & $\mathrm{mg} / \mathrm{L}$ & $\mathrm{B}$ & 4 \\
\hline Cadmio & $\mathrm{mg} / \mathrm{L}$ & $\mathrm{Cd}$ & 0.2 \\
\hline Cianuro & $\mathrm{mg} / \mathrm{L}$ & $\mathrm{CN}$ & 1 \\
\hline Cobre & $\mathrm{mg} / \mathrm{L}$ & $\mathrm{Cu}$ & 3 \\
\hline Cromo hexavalente & $\mathrm{mg} / \mathrm{L}$ & $\mathrm{Cr}$ & 0.5 \\
\hline Cromo total & $\mathrm{mg} / \mathrm{L}$ & $\mathrm{Cr}$ & 10 \\
\hline Manganeso & $\mathrm{mg} / \mathrm{L}$ & $\mathrm{Mn}$ & 4 \\
\hline Mercurio & $\mathrm{mg} / \mathrm{L}$ & $\mathrm{Hg}$ & 0.02 \\
\hline Niquel & $\mathrm{mg} / \mathrm{L}$ & $\mathrm{Ni}$ & 4 \\
\hline Plomo & $\mathrm{mg} / \mathrm{L}$ & $\mathrm{Pb}$ & 0.5 \\
\hline Sulfatos & $\mathrm{mg} / \mathrm{L}$ & $\mathrm{SQ} \mathrm{f}^{2}$ & 1000 \\
\hline Sulfuros & $\mathrm{mg} / \mathrm{L}$ & $\mathrm{S}$ & 5 \\
\hline Zinc & $\mathrm{mg} / \mathrm{L}$ & $\mathrm{Zn}$ & 10 \\
\hline Nitrógeno Amoniacal & $\mathrm{mg} / \mathrm{L}$ & $\mathrm{NH}$ & 80 \\
\hline pH & $\mathrm{unidad}$ & $\mathrm{pH}$ & $6-9$ \\
\hline Sólidos Sedimentables & $\mathrm{m} / \mathrm{L} / \mathrm{h}$ & $\mathrm{S} . \mathrm{S}$ & 8.5 \\
\hline Temperatura & ${ }^{\circ} \mathrm{C}$ & $\mathrm{T}$ & $<35$ \\
\hline
\end{tabular}

(1) La aplicación de estos parámetros a cada actividad económica por procesos productivos, será precisada en el reglamento de la presente norma tomando

Fuente: Diario El Peruano: Anexo N² del D.S. No021-2009 - Vivienda

\section{Formas de bajar la contaminación de las aguas residuales de la industria textil}

\section{Reducción en la fuente}

Existen variables que deben ser determinadas y evaluadas en el proceso de teńido de textil tales como caracterizar las descargas líquidas de cada operación, mantener un control de inventario y revisión de productos tóxicos, actualizar hojas técnicas de los productos químicos utilizados, sus instrucciones de manejo y sus características ecológicas, también evaluar periódicamente posibilidad de 
disminuir consumos de agua, de reactivos químicos y de la energía, y por último evaluar periódicamente el reemplazo de productos químicos por otros menos peligrosos para el ambiente.

Se ha planteado una gran variedad de estrategias para reducir la contaminación en la fuente, entre ellas se cuentan:

- Buenas prácticas de operación

- Sustitución de productos químicos

- Conservación del agua

- Reciclo/reuso/recuperación

La primera de ellas es parte de una buena gestión de operaciones al interior de la planta, e incluye la modificación de los procedimientos realizados en las operaciones productivas. Las otras apuntan más bien al uso de tecnologías limpias. Se definen como tecnologías limpias aquellos procesos que contribuyen a hacer más eficientes los métodos de producción, mediante el ahorro de energía y materias primas y la reducción de emisiones contaminantes al aire, agua y suelo.

\section{Recuperar: Reuso y reciclaje}

La mayoría de empresas en la industria textil recupera calor de las aguas residuales y para esto se puede usar pozos que almacenan por un tiempo los efluentes con algún contenido de calor antes de pasar por intercambiadores de calor de esta manera el agua entrante a la tintorería es aproximadamente de $35-50^{\circ} \mathrm{C}$ lo que ayuda a disminuir los tiempos de proceso y ahorrar en vapor.

Las aguas de enfriamiento indirecto que no están en contacto con el material textil ni con reactivos químicos pueden ser recolectadas y reusadas en forma di- recta. Estas aguas provienen de los intercambiadores de las máquinas de teñido por agotamiento y se pueden almacenar en un tanque o pozo de agua caliente $y$ reusarse en algunas operaciones de teñido, blanqueo, lavados, etc., donde se requiera agua tibia o caliente.

\section{Tratar: Planta de tratamiento de efluen- tes centralizado}

Los sistemas de tratamiento de efluentes permiten disminuir el nivel de contaminantes fuera del proceso y antes de descargarlos al sistema de alcantarillado o a algún río. El tipo de sistema a utilizar dependerá de las condiciones locales y de una serie de criterios de selección, como costos de inversión, operación y mantenimiento, eficiencia de remoción y rentabilidad, espacio disponible, personal especializado y de los estándares de valores máximos admisibles (VMA) de los efluentes que son vertidos a la red de alcantarillado.

Los procesos de tratamiento de aguas residuales pueden incluir tratamientos preliminares para remover sólidos gruesos y arena, tratamientos fisicoquímicos para remover sólidos sedimentables, materia orgánica, etc., tratamiento interno con rejas gruesas, sedimentación, regulación de flujo, ajuste de $\mathrm{pH}$ y temperatura; sistemas de filtración, tratamiento físico químico y tratamiento biológico.

Algunas empresas establecidas en el valle Lurín o Chincha que descargan sus efluentes a ríos tienen dentro de su sistema de gestión el tratamiento centralizado de las aguas residuales antes de ser vertidas, el cual incluye: tanque de neutralización, desarenado, sedimentación, torre de 
enfriamiento, floculación, filtración, microfiltración, osmosis inversa entre otros.
En la Tabla 2 se muestra los diferentes tratamientos para los efluentes textiles.

Tabla 2

Relación entre los tratamientos y su respectiva reducción de las descargas

\begin{tabular}{|c|c|c|}
\hline Proceso & Tratamiento de efluentes & Reducción de carga orgánica \\
\hline Lodos activados de carga reducida & Varios procesos & $\begin{array}{l}\text { 80\% (compuestos } \\
\text { Biodegradables) }\end{array}$ \\
\hline $\begin{array}{l}\text { Tratamiento físico, químico y } \\
\text { Biológico }\end{array}$ & Varios procesos & $\begin{array}{l}90 \% \text { (compuestos } \\
\text { Biodegradables + Compuestos } \\
\text { No biodegradables). }\end{array}$ \\
\hline $\begin{array}{l}\text { Tratamiento con técnica de } \\
\text { membrana }\end{array}$ & $\begin{array}{l}\text { Tintura, desencolado, } \\
\text { desengrasado }\end{array}$ & Hasta un $100 \%$ \\
\hline $\begin{array}{l}\text { Tratamiento y recuperación de } \\
\text { aguas residuales que contienen } \\
\text { pasta de pigmento }\end{array}$ & Pasta de pigmentos & $90 \%$ \\
\hline $\begin{array}{l}\text { Tratamiento anaeróbico de las } \\
\text { pastas de estampación y baños de } \\
\text { tintura }\end{array}$ & Tintura y estampación & $50-90 \%$ \\
\hline $\begin{array}{l}\text { Tratamiento de oxidación } \\
\text { química }\end{array}$ & Varios procesos & $70-85 \%$ \\
\hline $\begin{array}{l}\text { Tratamiento de floculación/ } \\
\text { precipitación }\end{array}$ & Varios procesos & $40-50 \%$ \\
\hline
\end{tabular}

Fuente: Salazar M. (2013)

Separar ciertas aguas residuales para tratamiento individual o reuso:

Valldeperas, Lis y Navarro presentaron en el año 2009 la solicitud de patente de invención que se refiere a un "Procedimiento de teñido de fibras textiles celulósicas y sus mezclas y poliéster y sus mezclas por medio de baños de teñido reciclado", con colorantes reactivos y directos para las fibras celulósicas y colorantes dispersos para el poliéster, reciclando tanto el agua ya utilizada en anteriores teñidos, así como todos los productos au- xiliares y químicos que se han agregado y no han sido absorbidos por el sustrato textil, además de los restos de colorantes dispersos que no se hayan agotado en los ciclos de teñido anteriores.

En el XX Congreso la Federación Latinoamericana de Químicos Textiles (FLAQT), realizado en nuestra capital en noviembre del 2010, la empresa brasileña Golden Química, presentó como primicia a nivel internacional la investigación sobre la reutilización de baños del teñido del algodón con colorantes reactivos con 
el asesoramiento de Valldeperas, destacado profesor investigador del INTEXTER-UPC. A partir del año 2012 lanzaron a nivel de producción el proceso DYE $\mathrm{CLEAN}^{\circledR}$ el cual fue patentado por ellos.

\section{Discusión}

Como se ha visto, el agua puede ser contaminada en diferentes puntos de la cadena de producción. Las aguas residuales procedentes de procesos de tintura y acabados representan el $80 \%$ del total de aguas residuales. El agua usada en el procesamiento de las fibras representa el $12 \%$, y el restante $8 \%$ procede de otros eslabones de la cadena de suministro.

La mayoría de las poblaciones humanas están expuestas a una variedad considerable de sustancias tóxicas. Los monitoreos biológicos han llevado a estudiar diferentes ocupaciones para explorar sus riesgos en la salud. Dönbak et al. (2007) evaluaron el posible riesgo genotóxico para los trabajadores de la industria textil, quienes se exponen a una amplia variedad de químicos como colorantes textiles, agentes blanqueadores, ácidos, álcalis y sales. En los resultados de su estudio, los autores revelan que existe un riesgo de genotoxicidad en dichos trabajadores.

Por otro lado, algunos compuestos químicos orgánicos pueden ser absorbidos y utilizados por algunas plantas, tales como: el melón, el rábano y la papa. Los efluentes textiles pueden reducir la germinación de las semillas y el crecimiento temprano de algunos vegetales (Zhou, 2001 y Rehman et al., 2008).

En los efluentes textiles se pueden encontrar metales como: arsénico, cadmio, cromo, cobalto, cobre, manganeso, mercurio, níquel, plata, titanio, zinc, estaño y plomo. Muchos de esos metales se generan durante el proceso de teńido.

En la Tabla 3 se muestran algunos metales presentes en diferentes clases de colorantes.

Tabla 3

Metales tipicos encontrados en colorantes

Clase de colorante Metales

\begin{tabular}{ll}
\hline \hline Directo & Cobre \\
Reactivo & Cobre y níquel \\
Ácido & Cobre, cromo, cobalto \\
Pre metalizado & Cobre, cromo, cobalto \\
Mordante & Cromo \\
\hline
\end{tabular}

Fuente: Cortázar, A. et al. (2005)

Teniendo en cuenta todos los aspectos negativos del impacto medioambiental de la industria textil, en los últimos años se vienen desarrollando alternativas a las formas de producción convencionales para poder lograr productos textiles menos contaminantes.

\section{Conclusiones}

Cada vez consumimos más cantidad de ropa y esta, a su vez, cada vez dura menos en nuestros armarios. Es un fenómeno llamado "fast fashion" y está causando estragos en nuestro planeta. Sin embargo, con el aumento del consumo también está aumentando la conciencia sobre el impacto que este tiene sobre el planeta. Greenpeace, a través de su campaña Detox, insta a las grandes compañías textiles que dejen de utilizar en sus prendas elementos químicos contaminantes que puedan tener efectos perjudiciales para la salud de la humanidad y de todo el planeta. 
El consumidor también ha evolucionado con los años, y empieza a interesarse por la composición de las prendas que viste. No solo le importa si el artículo puede lavarse o no a mano, sino que quiere conocer el origen de la materia prima, de la hilatura, del tintado y de la fabricación del tejido para saber si el producto es respetuoso con el entorno y con las personas que lo han elaborado. Si la etiqueta no lo pone fácil (a veces hay que descifrar su contenido), el ciudadano puede averiguar además si la empresa que ha confeccionado esa prenda posee alguna certificación o sello de calidad como "Made in Green by Oeko-Tex" (anteriormente conocida como Oeko-tex Standard 100), la etiqueta ecológica con mayor reputación a nivel mundial para determinar si las prendas textiles contienen sustancias nocivas.
Si las proyecciones que ha dado las Naciones Unidas para el crecimiento de la población mundial se cumplen, en el ańo 2050 duplicaremos la población actual de habitantes, la demanda de prendas y textiles del hogar se duplicará y por tanto necesitaremos cultivar el doble de algodón, criar el doble de ovejas, extraer el doble de celulosa para fabricar fibras artificiales (regeneradas), extraer el doble del petróleo para obtener fibras sintéticas (poliéster, poliamida, etc.).

Lo mismo sucederá con la cantidad del recurso agua y energía, pero también se duplicará el volumen de las aguas residuales textiles si es que desde ya no empezamos a trabajar en la reducción en la fuente, la reutilización, el reciclaje para los procesos productivos textiles y en tratamientos de depuración más eficientes.

\section{Referencias}

Bermeo, M. (2016). Remoción de colorantes de efluente sintético de industria textil aplicando tecnología avanzada de electro coagulación. Perú: Revista del Instituto de investigación FIGMMG-UNMSM.

Carrera, E. (2016). Los retos sostenibilistas del sector textil. Terrassa. Universidad Politécnica de Cataluña.

Castells, X. E. (2012). Tratamiento y valorizacion energética de residuos. Madrid: Díaz de Santos.

Cortázar A., Coronel C., Escalante A., González C., (2005). Contaminación generada por colorantes de la industria textil. Universidad Autónoma del Estado de Hidalgo.

E. Gilparas.-Arbeláez et al. (2017). Tratamiento de aguas residuales de la industria textil mediante coagulación quimica acoplada a procesos fenton intensificados con ultrasonido de baja frecuencia. Colombia: Revista Ingeniería y desarrollo de la Universidad Escuela de Administración Finanzas y tecnología (EAFIT).

Gutiérrez, M., Crespi, M., Lopez, V., \& Valldeperas, J. (2006). Reutilización de aguas residuales decoloradas mediante un tratamiento electroquimico. Intexter (Instituto de In- 
vestigación Textil y Cooperación Industrial de Terrassa, Universidad Politécnica de Cataluña).

Lopez-Grimau, V., \& Crespi Rosell, M. (2015). "Gestión de los efluentes de la industria textil". Buenos Aires, Argentina: Instituto Nacional de Tecnología Industrial, INTI.

López N. Tratamiento de agua superficial para la industria Textil. Congreso FLAQT XXI - 2013 Medellín Colombia

Nava, G. (2012). Cómo mejorar los problemas con Sedapal. Perú: CERTINTEX.

Peruano, El (2014). Normas legales. Perú: Ministerio del ambiente.

Rodríguez, J. (2005). Sistema de recuperación de agua residual textil mediante nanofiltración y ozonación para reuso en teñido-Edición única. México: Instituto tecnológico y de estudio superiores de Monterrey.

Sala, G. y Condorhuaman, C., Huella de carbono de la industria textil. Revista Peruana de Quimica e Ingeniería. Quimica. Vol 12 (2): 25-28, marzo 2014

Salazar, M. (2013). Técnicas disponibles paras el tratamiento de aguas residua- les textiles. Colombia: XXI Congreso Latinoamericano de Química Textil.

L. Salazar-Crespi et al. (2009). Tratamiento de aguas residuales textiles mediante un biorreactor de membrana. Perú: Revista Ingeniería y Desarrollo de la Universidad del Norte.

Tiedemann, K. (2013). Aguas residuales de la industria textil. Lo que debemos saber. Perú: Asociación Peruana de Técnicos Textiles (APTT).

Villegas Marín, C., \& González Monroy, B. (2013). FIBRAS TEXTILES NATURALES SUSTENTABLES Y NUEVOS HÁBITOS DE CONSUMO. Revista Legado de Arquitectura y Diseño, (13), 31-45.

WWAP. (2009). Informe de las Naciones Unidas sobre el desarrollo de los recursos hídricos en el mundo 2009, Reporte 3: Agua en un mundo cambiante. ONU. París: UNESCO y Londres: Earthscan.

WWAP (Programa Mundial de Evaluación de los Recursos Hídricos de las Naciones Unidas). 2017. Informe Mundial de las Naciones Unidas sobre el Desarrollo de los Recursos Hídricos 2017. Aguas residuales: El recurso desaprovechado. París, UNESCO. 